\title{
MUTATION OF KNOTS
}

\author{
C. KEARTON
}

(Communicated by Haynes R. Miller)

\begin{abstract}
In general, mutation does not preserve the Alexander module or the concordance class of a knot.
\end{abstract}

For a discussion of mutation of classical links, and the invariants which it is known to preserve, the reader is referred to [LM, APR, MT]. Suffice it here to say that mutation of knots preserves the polynomials of Alexander, Jones, and Homfly, and also the signature. Mutation of an oriented link $k$ can be described as follows. Take a diagram of $k$ and a tangle $T$ with two outputs and two inputs, as in Figure 1.

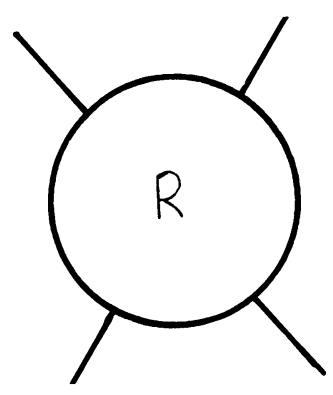

FIGURE 1

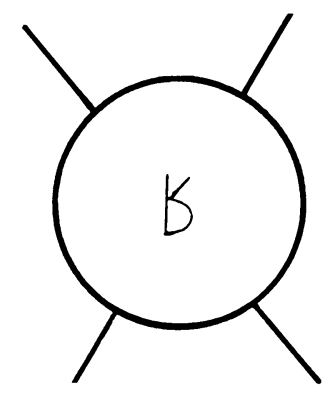

FIGURE 2

Rotate the tangle about the east-west axis to obtain Figure 2, or about the north-south axis to obtain Figure 3, or about the axis perpendicular to the paper to obtain Figure 4. Keep or reverse all the orientations of $T$ as dictated by the rest of $k$. Each of the links so obtained is a mutant of $k$.

The reverse $k^{\prime}$ of a link $k$ is obtained by reversing the orientation of each component of $k$. Let us adopt the convention that a knot is a link of one component, and that $k+l$ denotes the connected sum of two knots $k$ and $l$.

Lemma. For any knot $k$, the knot $k+k^{\prime}$ is a mutant of $k+k$.

Received by the editors September 16, 1987.

1980 Mathematics Subject Classification (1985 Revision). Primary 57M25. 


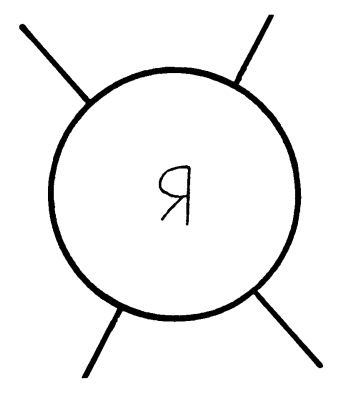

Figure 3

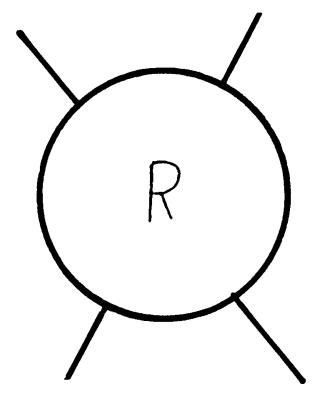

FIGURE 4

Proof. Shrink one of the summands in $k+k$ to a small knot, and arrange a diagram of $k+k$ to have a tangle as in Figure 5. Rotate about the axis perpendicular to the page, to obtain Figure 6, which represents $k+k^{\prime}$. Note that whatever convention we make about orientations, we always obtain $k+k^{\prime}$. Q.E.D.

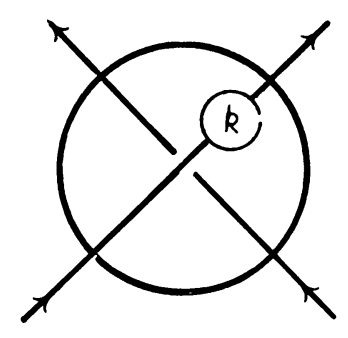

Figure 5

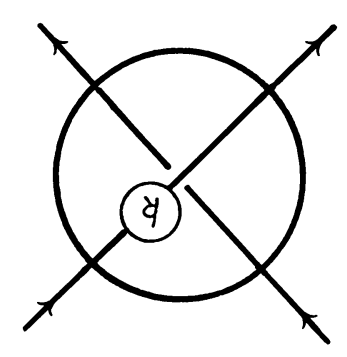

FIGURE 6

By a result of C. Livingston [L], there exist knots $k$ which are not concordant to their reverses $k^{\prime}$. It follows at once that $k+k$ is not concordant to $k+k^{\prime}$, and hence that mutation does not preserve the concordance class in general. I should like to thank Cameron Gordon for reminding me of Livingston's result.

In $[\mathrm{K}]$ there is an example of a knot $k$, in fact the pretzel knot $(25,-3,13)$, whose Steinitz-Fox-Smythe row ideal class $\rho$ does not satisfy $\rho^{2}=1$. The row ideal class of $k^{\prime}$, as pointed out in [K], is $\tau$, the column ideal class of $k$. Of course, $\rho \tau=1$, and so we see that the row ideal class of $k+k$ is $\rho^{2} \neq 1$, whereas the row ideal class of $k+k^{\prime}$ is $\rho \tau=1$. Thus we have an example in which the knot module of $k+k$ is not isomorphic to that of $k+k^{\prime}$. Another example can be obtained from [BHK, $\S 4$ ], and other examples can be found using $[B]$ and number theory tables. 


\section{ACKNOWLEDGMENT}

I wish to thank the Max-Planck-Institut für Mathematik, where this paper was written, for their hospitality. Also I wish to acknowledge the support of the Royal Society through their European Science Exchange Programme, and of the SERC through a travel grant.

\section{REFERENCES}

[APR] R. P. Anstee, J. H. Przytyck, and D. Rolfsen, Knot polynomials and generalized mutation, Topology Appl. (to appear).

[B] E. Bayer, Unimodular Hermitian and skew-Hermitian forms, J. Algebra 74 (1982), 341-373.

[BHK] E. Bayer, J. A. Hillman, and C. Kearton, The factorization of simple knots, Math. Proc. Cambridge Philos. Soc. 90 (1981), 495-506.

[K] C. Kearton, Noninvertible knots of codimension 2, Proc. Amer. Math. Soc. 40 (1973), 274-276.

[LM] W. B. R. Lickorish and K. C. Millett, A polynomial invariant of oriented links, Topology 26 (1987), 107-141.

[L] C. Livingston, Knots which are not concordant to their reverses, Quart. J. Math. Oxford 34 (1983), 323-328.

[MT] H. R. Morton and P. Traczyk, Knots, skeins and algebras, preprint.

Max-Planck- Institut für Mathematik, Gottfried-Claren-Strasse 26, D-5300 Bonn 3, WEST GERMANY land

Current address: Department of Mathematics, University of Durham, Durham DH1 3LE, Eng- 